\title{
Special review issue
}

\author{
David G. Robinson
}

Received: 19 October 2010 / Accepted: 20 October 2010 /Published online: 5 November 2010

(C) Springer-Verlag 2010

In contrast to our first special number devoted to reviews, which contained articles on subjects from all classes of organisms, this second review number has only plantrelated articles. This was not purposely arranged: On the contrary, it merely reflected a back log of accepted reviews which coincidentally had plant themes. With two exceptions (Rolfe and Scholles; Leborgne-Castel et al.), which are concerned with quite different aspects of plant-microbe interactions, these articles have no common denominator.

As a scientist who 25 years ago was involved in the struggle to get the process of endocytosis accepted by the plant community, I now have a feeling of great satisfaction at the increasing numbers of publications dealing with this theme. The review of Leborgne-Castel et al. shows just how far we have come in this regard and makes it abundantly clear that clathrin-mediated endocytosis is integral to the responses made by plants towards friend (symbiotic organisms) and foe (pathogens). This is a most timely article offering the reader a glimpse into potential developments in the exciting and rapidly expanding field of plant pathogen interactions.

Perhaps not so well known is the fact that chlorophyll fluorescence increases upon pathogen attack. Since this can be measured non-invasively, it allows one to monitor the photosynthetic performance of the host plant during the whole of the life cycle of the pathogen. Moreover, as an imaging technique, it even possesses a resolution down to cellular and subcellular levels. After providing us with a concise theoretical background to the origins of chlorophyll

D. G. Robinson $(\square)$

HIP-Cell Biology, University of Heidelberg,

Im Neuenheimer Feld 230,

69120 Heidelberg, Germany

e-mail: david.robinson@urz.uni-heidelberg.de fluorescence, Rolfe and Scholes give us a nice overview of the potentials of this technique to detect infection prior to the appearance of outwardly visible symptoms.

Anyone who has read Klionsky's perceptive 2007 review on autophagy (Nat Rev Mol Cell Biol 8:931) is aware of the explosion in the field of autophagy since the beginning of this decade (numbers of PubMed cited papers rising from about 40 per year in 2000 to over 500 in 2006!). This has been due to the awareness that there are connections between autophagy and cancer and some neurodegenerative disorders. Research into autophagic processes in plants has not witnessed such a dramatic trend, but there have nevertheless been significant advances. In their review, Reumann et al. describe the salient features of endocytosis shared by all eukaryotic organisms, but highlight the specific plant-related processes of this process. A new aspect in this area is how signal transduction cascades, in particular related to nitrogen-starvation, seem to trigger the onset of autophagy.

If like me, you are over 60 years of age, you should have a bone density scan, because as Dayod et al. state, "in Australia, $50 \%$ of females and $33 \%$ of males over the age of 60 will have an osteoporotic bone fracture...". Considering that low calcium diets have also been linked to colon cancer and high blood pressure, there is sufficient reason when you get older to watch your diet as far as calcium is concerned. After dairy products, plants are the most important suppliers of calcium in our diet, so it is clear that it is medically worthwhile to improve the calcium availability from plant sources. Dayod et al. give a very balanced account of the, not-so-successful, attempts made so far in this direction, for example by genetically modifying or breeding plants to produce less calciumbinding substances such as oxalate or phytate, or by generating plants overexpressing tonoplast $\mathrm{Ca}^{2+}$-transporters. 
In addition, the authors provide us with an excellent overview of calcium transport processes and calcium distribution in the whole plant, knowledge of which is essential for the goal of "biofortification of plants with additional calcium".

As everyone, including animal researchers, knows Arabidopsis thaliana (thale cress) is the model plant system. The last 15 years have seen the publication of many excellent papers dealing with the phenotyping of numerous mutants, e.g., KNOLLE, KEULE at the level of embryo development. However, as Ingram in her article most cogently reports: Angiosperm seed development is a very complicated process requiring a careful and coordinated interplay during the development of three genetically different tissues: the embryo, the endosperm, and the seed coat. This is a key article for all who want to know more about the "sweet" (and not-so-sweet) secrets of seed development, not only in Arabidopsis!

All first-year students in botany (now plant sciences) learn that woody plants arise through the activity of a secondary meristem - vascular cambium. But, and here I quote from the article of Risopatron et al.: "Given the role that forests play in carbon sequestration and the importance of wood-based products to industry, it is perhaps surprising that we know so little about the cellular and molecular basis of wood production in trees." Therefore, I could think of no better reason to have an update on this subject. However, in presenting the most recent data on Arabidopsis mutants, which show defects in vascular development in Arabidopsis, I think that Risopatron et al. have given sufficient evidence that we are now well on the way to redressing the balance.

Tip growth in plants is studied either on root hairs or germinating pollen tubes. In both systems, a continuous flow of secretory vesicles to the tip is maintained by interactions between the cytoskeleton and molecular motors. Whereas the participation of actin filaments and myosin-type motors has been established, the role of microtubules and kinesins/dyneins is not at all clear. Cai and Cresti critically examine the evidence for microtubules and microtubule-based motors in pollen tubes and come to the conclusion that the jury is still out on this issue. 